\title{
CORRELAÇÃO ENTRE FORÇA DE PREENSÃO MANUAL, DISTÂNCIA DA PREGA PALMAR MÉDIA E CIRCUNFERÊNCIA DE ANTEBRAÇO.
}

\section{Correlation between handgrip strength, palmar medial fold distance and forearm circunference}

\author{
Bibiano Madrid ${ }^{1,2}$, Jeeser Alves de Almeida', Glauber Flores Silva ${ }^{3}$, Rafael dos Santos Cruz ${ }^{3}$, \\ Marcelo Magalhães de Sales ${ }^{1}$, Suliane Beatriz Rauber ${ }^{3}$ \\ ${ }^{1}$ Programa de Pós-graduação em Educação Física, Universidade Católica de Brasília (UCB), Taguatinga-DF, Brasil. \\ ${ }^{2}$ Laboratório de Avaliação Física e Treinamento (LAFIT-UCB). \\ ${ }^{3}$ Programa de Graduação em Educação Física - UCB.
}

Resumo: A força de preensão manual tem estado associada à mortalidade, limitações funcionais, incapacidade e estado nutricional. Porém, o dinamômetro sem ajuste de empunhadura, amplamente utilizado na literatura, poderia estar influenciando nos resultados obtidos. $\mathrm{O}$ objetivo do presente estudo foi correlacionar à força de preensão manual (FPM) em dinamômetro sem ajuste de empunhadura com a circunferência de antebraço e também com a distância da prega palmar média até a extremidade distal do dedo médio, verificando a influência desta última variável sobre a melhor empunhadura e sobre a utilização adequada ou não do dinamômetro de preensão manual sem ajuste para empunhadura em

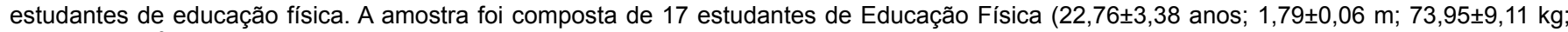
$23 \pm 2,32 \mathrm{~kg} / \mathrm{m}^{2}$ ), que foram submetidos a medidas antropométricas (massa corporal, estatura, e circunferência do antebraço) e teste de força de preensão manual, realizando duas tentativas (com dois minutos de intervalo entre elas). Foi utilizada análise descritiva através de média, desvio padrão, valores máximos e mínimos. Também foi empregada correlação de Pearson, adotando-se $p<0,05$ e o software utilizado foi o Instat 3.0 . A força de preensão manual $(51,65 \pm 6,23 \mathrm{kgf})$ se correlacionou significativamente com circunferência de antebraço $(27,68 \pm 1,84 \mathrm{~cm})(\mathrm{r}=0,72)$, mas não mostrou correlação com a distância da prega palmar média $(12,55 \pm 0,64 \mathrm{~cm})(r=0,46)$. Verificou-se correlação significativa entre a força de preensão manual e circunferência de antebraço, confirmando a hipótese de que o maior perímetro de antebraço determinaria um maior nível de força de preensão manual. Em relação à força de preensão manual e distância da prega palmar média não se observou correlação significativa, não confirmando a hipótese de que o tamanho da mão influenciaria diretamente nos resultados de preensão manual em avaliação com dinamômetro sem ajuste de empunhadura.

Palavras-chave: Força da Mão, Dinamômetro de Força Muscular, Mãos, Força Muscular.

\begin{abstract}
The handgrip has been associated with mortality, functional limitations, and disability status. However, the dynamometer without adjustment handle, widely used in literature, could be influencing the results The aim of this study was to correlate the handgrip (FPM) on dynamometer without adjustment of grip with the circumference of the forearm and also with the away from the middle palmar crease to the distal end of the middle finger, checking the influence of that variable on the best grip and the proper use or otherwise of the handgrip dynamometer without adjustment for grip in physical education students. The sample consisted of 17 physical education students $(22.76 \pm 3.38$ years, $1.79 \pm 0.06$ $\mathrm{m}, 73.95 \pm 9.11 \mathrm{~kg}, 23 \pm 2.32 \mathrm{~kg} / \mathrm{m}^{2}$ ), who underwent measures Anthropometric (body weight, height and circumference of the forearm) and test of grip strength, making two attempts (with two minute interval between them). Descriptive analysis was used as mean, standard deviation, maximum and minimum values. Was also used Pearson correlation, adopting $p<0.05$ and the software used was Instat 3.0. The grip strength (51.65 \pm 6.23 $\mathrm{kg})$ correlated significantly with forearm circumference $(27.68 \pm 1.84 \mathrm{~cm})(\mathrm{r}=0.72)$, but showed no correlation with the distance from the middle palmar crease $(12.55 \pm 0.64 \mathrm{~cm})(r=0.46)$. There was significant correlation enters the grip strength and forearm circumference, confirming the hypothesis that the largest circumference of forearm determine a higher level of grip strength. Regarding grip strength and distance from the palmar crease mean there was no significant correlation, not confirming the hypothesis that the size of the hand directly influence the results of handgrip dynamometer in the evaluation without adjustment for grip.
\end{abstract}

Keywords: Hand Strength, Muscle Strength Dynamometer, Hand, Muscle Strength.

Aceito em 17/02/2010 - Rev. Educ. Fís. 2010 - 15-19. Rio de Janeiro - RJ - Brasil

INTRODUÇÃO

A mão é o segmento terminal do membro superior, continuação do punho e que termina distalmente com os dedos. Seu limite proximal é dado por um plano horizontal que passa pelo pisiforme e pelo escafóide. Ela tem como elementos extraordinários, a preensão e a sensibilidade, comandadas pelo córtex 
cerebral. Descrevendo anatomicamente podemos dividi-la em duas regiões: ventral e dorsal. $\mathrm{Na}$ parte ventral encontram-se três pregas cutâneas, que são conseqüências de movimentos fisiológicos, como flexão, extensão, e oponência. Estas pregas têm como referência uma figura em formato da letra "M" com sua base voltada para o lado ulnar. A prega palmar média (figura 1) está localizada cerca de $2,5 \mathrm{~cm}$ distal do arco arterial profundo e $1,5 \mathrm{~cm}$ distal do arco palmar superficial( ${ }^{(1)}$.

A força de preensão manual (FPM), mensurada por dinamometria, é uma importante variável relacionada ao crescimento e desenvolvimento biológico, que sofre drásticas alterações morfológicas com o passar dos anos, especialmente na segunda década de vida ${ }^{(2)}$. A FPM tem sido um importante marcador da força total do indivíduo, sendo empregada em testes de aptidão física. Além disso, Barbosa et al. ${ }^{(3)}$ associaram os níveis de FPM à mortalidade, limitações funcionais, incapacidade e estado nutricional.

Uma das variáveis que influenciam os níveis de força, especialmente a FPM, é a área de secção transversa da musculatura do antebraço, que estaria relacionada com o desenvolvimento global de força $a^{(4)}$. Nesse sentido, Franchini et al. ${ }^{(4)}$ demonstraram forte correlação entre a FPM e a circunferência do antebraço em lutadores de jiu-jítsu $(r=0,724)$. Já Bertuzzi et al. ${ }^{(5)}$ realizaram um estudo relacionando o nível de FPM com variáveis antropométricas e observaram uma forte correlação somente com a circunferência do antebraço $(r=0,74)$. Além deste, Esteves et al. ${ }^{(6)}$ atribuem melhores performances da FPM as proporções da mão dos avaliados que tinham a melhor empunhadura da distância da palma da mão até a articulação entre a primeira e segunda falanges, necessitando de mais estudos para confirmações.

A utilização de dinamômetro com empunhadura ajustável para força de preensão manual necessita de recursos financeiros maiores e, em função disto, modelos de dinamômetros sem esse artifício de ajuste são amplamente utilizados na literatura. Sendo assim, esse tipo de limitação de equipamento pode influenciar nos resultados obtidos e conseqüentemente numa avaliação incorreta do que se pretende mensurar.

Logo, o objetivo do presente estudo foi correlacionar a FPM em dinamômetro sem ajuste de empunhadura com a circunferência de antebraço (CA) e com a distância da prega palmar média até a extremidade distal do dedo médio (DPPM), verificando a influência desta última variável sobre a melhor empunhadura e sobre a utilização adequada ou não do dinamômetro de preensão manual sem ajuste para empunhadura em estudantes de Educação Física.

\section{METODOLOGIA}

A amostra foi composta por 17 voluntários do sexo masculino (22,76 \pm 3,38 anos; 1,79 \pm 0,06 m; 73,95 \pm $9,11 \mathrm{~kg} ; 23 \pm 2,32 \mathrm{~kg} / \mathrm{m}^{2}$ ), acadêmicos do curso de Educação Física, recrutados por conveniência. Antes de iniciar as coletas todos os voluntários assinaram o termo de consentimento livre e esclarecido.

Inicialmente a massa corporal foi mensurada em uma balança eletrônica (Toledo 2096 PP, com precisão de 50 gramas) e a estatura foi medida em um estadiômetro (Sanny Standard, com escala de dois $\mathrm{mm}$ ). A circunferência do antebraço (CA) foi mensurada no antebraço dominante, com o braço relaxado e estendido ao longo do corpo, considerando CA a porção do membro que possuísse o maior perímetro. A prega palmar média foi tomada no arco arterial profundo (figura 1). A DPPM foi medida da prega palmar média até a extremidade distal do dedo médio da mão dominante. Essa variável foi mensurada com o cotovelo flexionado, dedos estendidos e a mão em posição supina (Trena antropométrica Sanny, com escala de 0,10 mm).
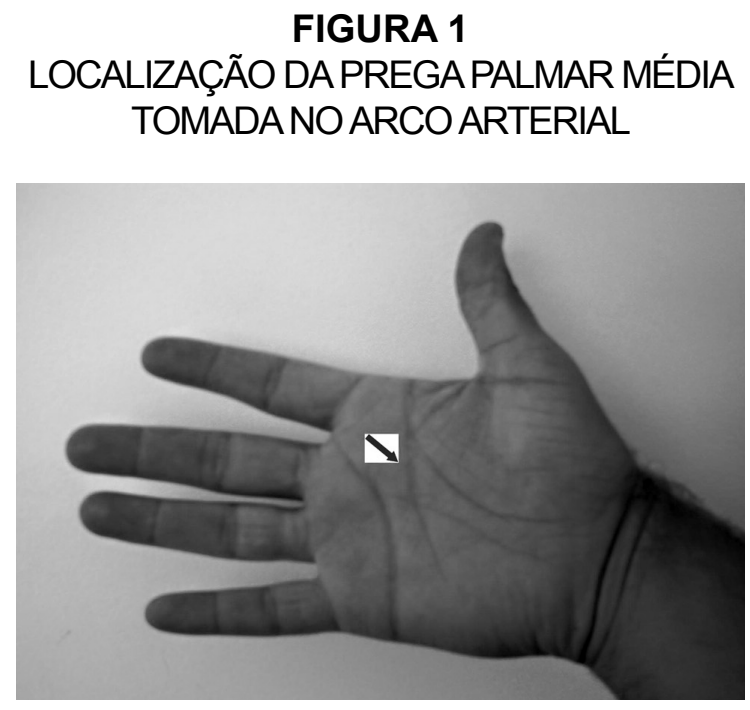

Para aferição da força de preensão manual (FPM) o instrumento utilizado foi um dinamômetro (Crown - calibrado com escala de 0 a $100 \mathrm{Kgf}$ ), onde o voluntário ficava na posição em pé, com o braço dominante estendido ao longo do corpo empunhando o dinamômetro. Ao sinal do avaliador o voluntário realizava uma preensão máxima voluntária (conforme descrito pelo fabricante). 
Após dois minutos o procedimento era repetido, foi adotado como a FPM o maior valor absoluto das duas tentativas para cada indivíduo, conforme utilizado por Madrid et al. ${ }^{(7)}$.

Para descrição dos dados foi utilizada análise descritiva através de média e desvio padrão (DP) e valores máximos e mínimos. Foi empregada correlação de Pearson e o software Instat versão 3.0 para verificar significância, adotando-se $p<0,05$.

\section{RESULTADOS}

Os valores de força de preensão manual (FPM), distância da prega palmar média (DPPM) e circunferência do antebraço (CA) estão apresentadas na TABELA 1 em média, desviopadrão (DP), mínimo e máximo.

TABELA 1

RESULTADOS PRINCIPAIS EM MÉDIA, DESVIO PADRÃO

\begin{tabular}{cccc}
\hline & $\begin{array}{c}\text { FPM } \\
(\mathrm{kgf})\end{array}$ & $\begin{array}{c}\text { DPPM } \\
(\mathrm{cm})\end{array}$ & $\begin{array}{c}\text { CA } \\
(\mathrm{cm})\end{array}$ \\
\hline Média & 51,65 & 12,55 & 27,68 \\
DP & 6,23 & 0,64 & 1,84 \\
Mínimo & 44,5 & 11,0 & 24,2 \\
Máximo & 69,0 & 13,6 & 31,5 \\
\hline
\end{tabular}

Força de preensão manual (FPM), distância da prega palmar média (DPPM) e circunferência do antebraço (CA).

Na TABELA 2 estão apresentados os valores de correlação de Pearson entre FPM, DPPM e CA $(p<0,05)$, onde se obteve correlação significativa somente entre a FPM e CA $(r=0,72)$.

TABELA 2

MATRIZ DE CORRELAÇÕES ENTRE FORÇA DE PREENSÃO MANUAL (FPM), DISTÂNCIA DA PREGA PALMAR MÉDIA (DPPM) E CIRCUNFERÊNCIA DE ANTEBRAÇO (CA).

\begin{tabular}{ccc}
\hline & DPPM & CA \\
\hline FPM & $r=0,46 \quad r=0,72^{*}$ \\
\hline & 'Diferença significativa $(p<0,05)$
\end{tabular}

\section{DISCUSSÃO}

O objetivo do presente estudo foi correlacionar a força de preensão manual (FPM) utilizando um dinamômetro sem ajuste de empunhadura, com a circunferência de antebraço (CA) e a distância da prega palmar média até a extremidade distal do dedo médio (DPPM) em estudantes de educação física. Encontramos correlação significativa para a relação da FPM com a CA $(r=0,72)$, mas não obtivemos correlações significativas entre a FPM e a DPPM $(r$ $=0,46)$. $O$ teste de preensão manual mede a força da musculatura flexora dos dedos da mão e pode ser aplicado em ambos os sexos, com boa fidedignidade $(r=0,90)^{(8)}$.

Nossos resultados de FPM $(51,65 \pm 6,23 \mathrm{kgf})$ são similares aos de Franchini et al. ${ }^{(4)}$ em lutadores de jiu-jítsu (54,2 $\pm 6,7$ kgf), porém, superiores aos de Pasetti et al. ${ }^{(9)}$ com mulheres obesas submetidas a 17 semanas de treinamento de corrida em piscina profunda (31,52 $\pm 6,55 \mathrm{kgf})$, assim como os de Madrid et al. ${ }^{(7)}$ em idosas com idade entre 60 e 69 anos e entre 70 e 79 anos praticantes de musculação $(26,88$ $\pm 4,19 \mathrm{kgf}$ e 25,06 $\pm 3,11 \mathrm{kgf}$, respectivamente).

Watts et al. ${ }^{(10)}$ verificaram que a FPM de escaladores competitivos não diferiram entre finalistas e semifinalistas de uma etapa da Copa do Mundo de Escalada Esportiva Indoor. Contudo, comparados a sujeitos sedentários, os valores apresentados por esses atletas eram superiores demonstrando de forma prática que o método de avaliação da FPM caracteriza, com confiança, os níveis de condicionamento e de força de membros superiores.

Moreira et al. ${ }^{(11)}$, utilizando atletas de jiujítsu, correlacionaram à preensão manual com a circunferência de antebraço, encontrando uma baixa correlação $(r=0,54)$, ao passo que, Franchini et al. ${ }^{(4)}$ com a mesma população encontraram correlação idêntica a nossa $(r=0,72)$. Os valores de CAdo nosso estudo em estudantes de Educação física (27,68 \pm $1,84 \mathrm{~cm}$ ) foram similares aos escaladores de elite $(26,6 \pm 1,2 \mathrm{~cm})$, recreacionais $(26,5 \pm 0,9 \mathrm{~cm})^{(12)} \mathrm{e}$ judocas $(28,6 \pm 1,9)^{(13)}$. Em contrapartida inferiores aos de culturistas $(31,85 \pm 1,83 \mathrm{~cm})^{(14)}$, possivelmente em função de treinamentos específicos e probabilidade de benefícios advindos de recursos ergogênicos, comumente utilizados nessa população.

Em um estudo similar, porém em outra população, autores analisaram as dimensões antropométricas da mão e a força de preensão palmar em 189 jogadores de handebol e basquetebol de 10 a 17 anos, e não foi observada correlação significativa ${ }^{(15)}$. Os autores asseguraram que o tamanho da mão não exerce influência na força de preensão. Eles utilizaram 
três valores antropométricos relacionados à mão: extensão, comprimento dos dedos e perímetro da mão, a força de preensão foi medida com a mão dominante, usando-se o dinamômetro Lafayette $\AA$. Concluíram que os parâmetros antropométricos gerais, como peso e altura, foram mais importantes para dimensionar a força máxima de preensão palmar. Em outro estudo, Ruiz-Ruiz et al. ${ }^{(16)}$, constataram que o tamanho da mão exercia influências na força de preensão em mulheres adultas.

Não haver correlação significativa entre os valores de DPPM e a FPM nos mostra que possivelmente a utilização de um dinamômetro de preensão manual sem ajustes não seja um fator que altere os resultados de maneira direta, pois a hipótese de quanto maior a mão do avaliado maior a força gerada foi rejeitada no presente estudo. Os valores de FPM e CA encontrados em nosso estudo estão de acordo com o documentado na literatura. Já quanto a DPPM não foram encontrados valores de referência.

A fim de ampliar o conhecimento acerca dos achados no presente artigo, sugerem-se estudos com amostras maiores, diferentes populações, bem como a comparação dos valores de FPM obtidos em dinamômetros com e sem ajustes de empunhadura.

\section{CONCLUSÃO}

A força de preensão manual (FPM) foi positivamente correlacionada com a circunferência de antebraço (CA), confirmando a hipótese de que quanto maior o perímetro do antebraço, maiores são os níveis de FPM. Já para correlação entre a FPM e DPPM não se observou correlação significativa, rejeitando assim a hipótese de que o tamanho da mão influenciaria diretamente nos resultados de preensão manual em avaliação com dinamômetro sem ajuste de empunhadura. $O$ que nos faz sugerir que para a população estudada o dinamômetro sem ajuste de empunhadura não interfere diretamente nos resultados de FPM.

\section{REFERÊNCIAS BIBLIOGRÁFICAS}

1. Mattar Junior R, Azze RJ. Semiologia da Mão. In: Publicação Oficial do Instituto de Ortopedia e Traumatologia do HC FMUSP. Atualização em Traumatologia do Aparelho Locomotor. São Paulo: 1999.
2. Giarolla RA, Figueiredo Junior AJ, Matsudo VKR. Análise da força da mão dominante em relação à mão não-dominante em escolares de 8 a 18 anos. Revista Brasileira de Ciência e Movimento 1991;5(1):31-39.

3. Barbosa AR, Souza JMP, Lebrão ML, Marucci MFN. Relação entre estado nutricional e força de preensão manual em idosos do município de São Paulo, Brasil: dados da Pesquisa SABE. Revista Brasileira de Cineantropometria \& Desempenho Humano 2006;8(1):37-44.

4. Franchini E, Takito MY, Pereira JNC. Freqüência cardíaca e força de preensão manual durante a luta de jiu-jítsu. EF Deportes 2003;9(65).

5. Bertuzzi RCM, Franchini E, Kiss MAPD. Análise da força e da resistência de preensão manual e as suas relações com variáveis antropométricas em escaladores esportivos. Revista Brasileira de Ciência e Movimento 2005;13(1):87-93.

6. Esteves AC, Reis DC, Caldeira RM, Leite RM, Moro ARP, Borges Junior NG. Força de preensão, lateralidade, sexo e características antropométricas da mão de crianças em idade escolar. Revista Brasileira de Cineantropometria \& Desempenho Humano 2005;7(2):69-75.

7. Madrid B, Souza LHR, Oliveira RJ. Contribuições do treinamento resistido para força de membros superiores em idosas. In: XXIX Simpósio Internacional de Ciências do Esporte, São Paulo-SP. A Globalização do esporte e da atividade física. Edição Especial da Revista Brasileira de Ciência e Movimento, Suplemento Especial 2006;14(4):260.

8. Marins JCB, Giannichi RS. Avaliação e prescrição de atividade física. Guia prático. 3 ed. Rio de Janeiro: Shape; 2003, p. 111.

9. Pasetti SR, Gonçalves A, Padovani CR. Corrida em piscina profunda para melhora da aptidão física de mulheres obesas na meia idade: estudo experimental de grupo único. Revista Brasileira de Educação Física Especial. 2006;20(4):297-304.

10. Watts PB, Martin DT, Durtschi S. Anthropometric profiles of elite male and female competitive rock climbers. Journal Sports Science 1993;11(2):113-117.

11. Moreira SR, Gulak A, Silva JU, Ronque VER, Silva KES. Correlação de variáveis antropométricas de membros superiores com a força de preensão manual em praticantes de jiu-jítsu. Motriz 2003;9(1):S147-148. 
12. Bertuzzi RCM, Franchini E, Kiss MAPD. Ajustes agudos da freqüência cardíaca e da preensão manual na prática da escalada esportiva indoor. Revista Mackenzie de Educação Física e Esporte 2004;3(3): 99-106.

13. Franchini E, Souza CEB, Urasaki R, Sauressig F, Oliveira RSF, Matheus L. Teste de resistência de força isométrica e dinâmica na barra com o judogi. In: III Congresso de La Asociación Española de Ciencias da La Actividad Física y el Deporte de La Universidad de Valencia, 2004.

14. Cyrino ES, Maestá N, Burini RC. Aumento de força e massa muscular em atletas de culturismo suplementados com proteína. Revista Treinamento Desportivo 2000;5(1):9-18.
15. Visnapuu M, Jürimäe T. Handgrip strength and hand dimensions in young handball and basketball players. $J$ Strength Cond Res 2007;21(3):923-929.

16. Ruiz-Ruiz J, Mesa JLM, Gutiérrez A, Castillo MJ. Hand Size Influences Optimal Grip Span in Women but not in Men. J Hand Surg 2002;27(5):897-901.

Endereço para correspondência:

UCB - LAFIT

QS 07, Lote 01, S/N, Bloco G, Sala 120 D.

Cep: 71966-700. Bairro Areal. Taguatinga-DF. Telefones: (61) 3356-9044.

Fax: (61) 3356-9350.

e-mail: bibiano.madrid@gmail.com 